\title{
Building oscillations bottom up: Elemental time scales of intracellular calcium dynamics
}

\author{
Rüdiger Thul ${ }^{1}$ and Martin Falcke ${ }^{1}$ \\ 1 Hahn-Meitner Institut, Abteilung Theorie, Glienicker Str. 100, D-14109 Berlin, \\ Germany thul@hmi.de \\ 2 Hahn-Meitner Institut, Abteilung Theorie, Glienicker Str. 100, D-14109 Berlin, \\ Germany falcke@hmi.de
}

Summary. We analyze the elemental time scale of intracellular calcium dynamics. It is determined by the time course of $\mathrm{Ca}^{2+}$ puffs, which represent the fundamental quantum of $\mathrm{Ca}^{2+}$ release from intracellular storage compartments. Since $\mathrm{Ca}^{2+}$ puffs are truly random, we propose a novel master equation and corresponding FokkerPlanck equations. Our results demonstrate that puff initiation can be mapped to an escape process. The stochastic fraction of puff periods is identified with mean first passage times. We find that the discrete character of release sites represents a necessary condition for puff initiation. A continuous modeling of the number of open channels does not allow $\mathrm{Ca}^{2+}$ puffs in the relevant part of the parameter space.

\section{Introduction}

The unraveling of molecular interactions has been providing intriguing insights into cellular organization during the last decades. On the one hand, qualitative results showed connections between formerly unassociated reactions. For instance, one of the molecular mechanisms that underlies cancer was identified to play a central role in Alzheimer's disease [22, 2]. On the other hand, the ongoing improvement in experimental techniques allowed quantitative investigations. They revealed that cells use a plethora of different amounts of molecules for a variety of tasks. Some pathways involve macroscopically large numbers of chemical agents, whereas others are controlled by only some tens of molecules. This discrepancy in the number of reactants attributes a twin role to fluctuations. They can be neglected in the former reactions, but play a pivotal role in the latter. Experiments on gene expression demonstrated that fluctuations can decide upon the phenotype leading to population heterogeneity $[23,19]$. For a general review on intracellular noise, see [26].

Modeling intracellular phenomena therefore demands an a priori choice of methods. As long as fluctuations are negligible, deterministic equations correctly capture the dynamics $[10,17]$. However, these approaches break down 
in the presence of noise. Comparisons between stochastic models and their deterministic counterparts have revealed that noise can induce a dynamical behavior that is not present in the absence of fluctuations. For instance, the MinCDE system only oscillates if the experimentally observed small number of interacting molecules is respected [16]. The deterministic equations decay to a fixed point.

The above examples all have in common that the total number of interacting molecules in the entire cell is small. However, fluctuations can also arise due to cellular heterogeneity. Steep concentration gradients may create very heterogeneous conditions within a single cell such that the conditions for a reaction to occur are given in a small part of the cell only. Since these gradients are typically transient we call this phenomenon dynamic compartmentalization. The number of interacting molecules in such a dynamic compartment may only be a tiny fraction of the molecules present in the whole cell. Then, fluctuations remain large and cannot be neglected, although the total number of molecules in the whole cell would allow for mean field behavior. That is the case with intracellular $\mathrm{Ca}^{2+}$ dynamics. The dynamic compartment is a single $\mathrm{Ca}^{2+}$ releasing channel cluster as is explained below.

The dynamics of the $\mathrm{Ca}^{2+}$ concentration in the cytosol of a cell is determined to a large degree by release and uptake of $\mathrm{Ca}^{2+}$ by intracellular storage compartments, in particular the endoplasmic reticulum (ER). Release is controlled by inositol-1,4,5-trisphosphate $\left(\mathrm{IP}_{3}\right)$ receptor channels $\left(\mathrm{IP}_{3} \mathrm{R}\right)$. They are arranged in clusters with a diameter of less than $100 \mathrm{~nm}$ that comprise between 1 and 40 channels and that are randomly distributed on the membrane of the ER with distances between 1-7 $\mu \mathrm{m}[30,21] . \mathrm{IP}_{3} \mathrm{Rs}$ have the important property that their open probability depends on the $\mathrm{Ca}^{2+}$ concentration in the cytosol. The details of this dependency will be discussed in section 2. A moderate increase in the cytosolic concentration - i.e. on the outside of the storage compartment - increases the opening probability.

The notion of an open probability goes along with the generally stochastic character of ion channels, i.e. a single $\mathrm{IP}_{3} \mathrm{R}$ channel opens and closes randomly $[14,35]$. Since the number of $\mathrm{Ca}^{2+}$ release channels per cluster is small, fluctuations still prevail in a cluster [7]. They lead to random release events called $\mathrm{Ca}^{2+}$ puffs. A puff is the spontaneous opening of channels of a single cluster and represents the elemental event of $\mathrm{Ca}^{2+}$ liberation. Puffs last from a few tens of milliseconds to a few hundred milliseconds and they cause a huge but strongly localized concentration rise. Many puffs can cooperate to build global phenomena covering the whole cell like waves of release or oscillations $[21,6]$, i.e. puffs constitute the fundamental building block of $\mathrm{Ca}^{2+}$ signals. We illustrate this concept with the initiation and the spreading of a $\mathrm{Ca}^{2+}$ wave. Assume that all channels are closed and the cell is in its resting state. Fluctuations due to the random association and dissociation of $\mathrm{Ca}^{2+}$ and $\mathrm{IP}_{3}$ to the $\mathrm{IP}_{3} \mathrm{Rs}$ eventually lead to a $\mathrm{Ca}^{2+}$ puff. $\mathrm{Ca}^{2+}$ is liberated from the ER and diffuses to the surrounding clusters. There, it causes an increase of the open probability and may therefore induce channels of these clusters to open. That 
gives rise to another $\mathrm{Ca}^{2+}$ puff and release has propagated by one cluster distance. In that way a $\mathrm{Ca}^{2+}$ wave travels through the cell. However, there is no guarantee that $\mathrm{IP}_{3} \mathrm{R}$ channels at neighboring clusters open, because channel opening is a truly random event.

Indeed, fluctuations turned out to be necessary to observe any temporal or spatial structures in intracellular $\mathrm{Ca}^{2+}$ dynamics. That role of fluctuations has been established by two complementary approaches. On the one hand, oscillations that agree with experimental findings in stochastic simulations disappear in the deterministic limit of the simulated system [6]. On the other hand, a bifurcation analysis of a deterministic model for a single $\mathrm{IP}_{3} \mathrm{R}$ cluster has proofed that the local dynamics is non-oscillatory when realistic $\mathrm{Ca}^{2+}$ fluxes and gradients are incorporated [32, 33, 31]. The loss of oscillations results from the high $\mathrm{Ca}^{2+}$ concentrations at an open cluster. They lead to a saturation of all $\mathrm{Ca}^{2+}$ regulating processes and hence do not permit $\mathrm{Ca}^{2+}$ oscillations in deterministic models. Fluctuations drive the channel dynamics out of the saturated state and eventually reinstall oscillations. The strong localization of the $\mathrm{Ca}^{2+}$ liberation and the entailing large gradients around an open cluster create the dynamic compartmentalization mentioned above. Since the volume of the elevated $\mathrm{Ca}^{2+}$ concentration as well as the number of $\mathrm{IP}_{3} \mathrm{Rs}$ that experience this highly increased $\mathrm{Ca}^{2+}$ concentration is small, fluctuations remain important.

Given the vital part of $\mathrm{Ca}^{2+}$ puffs in intracellular $\mathrm{Ca}^{2+}$ dynamics and the importance of fluctuations, a stochastic description of a single cluster is the focus of the present report. We will apply it to the initiation of $\mathrm{Ca}^{2+}$ puffs, which represents the first step for any $\mathrm{Ca}^{2+}$ pattern. Our findings suggest that puff initiation can be mapped to an escape process from the resting state towards the first open channel. The mean first passage time corresponds to the stochastic part of the puff frequency. The mathematical description has to account for the integer number of open channels per cluster. A continuous $\mathrm{Ca}^{2+}$ model (using non-integer fractions of open channels) that incorporates realistic fluxes does not permit $\mathrm{Ca}^{2+}$ puffs for parameter values that agree with experimental data. The mean first passage times can be represented as an infinite series of exponentials. However, already the first terms in the expansion yield excellent convergence. That hints at a Poissonian character of puff initiation. Although noise is intrinsically multiplicative for intracellular $\mathrm{Ca}^{2+}$ dynamics, we provide evidence that additive noise may serve as a reasonable approximation.

We will introduce a $\mathrm{Ca}^{2+}$ model for an $\mathrm{IP}_{3}$ receptor channel cluster in the next section. It serves as input for a master equation in section 3 , from which we will derive two Fokker-Planck equations in section 4. Finally, we will employ these equations to characterize the initiation of $\mathrm{Ca}^{2+}$ puffs. 


\section{$2 \mathrm{Ca}^{2+}$ model}

The $\mathrm{IP}_{3}$ receptor channel is a tetramer the subunits of which have binding sites for $\mathrm{Ca}^{2+}$ and $\mathrm{IP}_{3}$. We implement a model for a single subunit that is based on ideas of De Young's and Keizer's [5]. They assume a subunit to possess three binding sites: an activating $\mathrm{Ca}^{2+}$ binding site, an inhibiting $\mathrm{Ca}^{2+}$ binding site and an activating $\mathrm{IP}_{3}$ binding site. The occupation of the binding sites controls the state of the subunit. When $\mathrm{IP}_{3}$ and $\mathrm{Ca}^{2+}$ are bound to their activating binding sites, a subunit is in the activated state. As soon as $\mathrm{Ca}^{2+}$ binds to the inhibiting binding site, a subunit is inhibited, independent of the state of the other binding sites. It can only be activated again upon dissociation of $\mathrm{Ca}^{2+}$ from the inhibiting binding site. Experiments have indicated that an $\mathrm{IP}_{3} \mathrm{R}$ channel is conducting when at least 3 subunits are activated [4, 35]. Random binding and unbinding of $\mathrm{Ca}^{2+}$ and $\mathrm{IP}_{3}$ and therefore random state changes of the receptor are the source of stochasticity of intracellular $\mathrm{Ca}^{2+}$ dynamics.

The number of open $\mathrm{IP}_{3} \mathrm{R}$ channels determines the $\mathrm{Ca}^{2+}$ flux from the ER to the cytosol. Since the release channels are tightly packed within a cluster, a relation between the number of channels in the cluster and cluster size exists. Consequently, we can map the number of open channels to the size of a conducting area (or volume) equal to the area occupied by all open channels. A change in the number of releasing $\mathrm{IP}_{3} \mathrm{R}$ channels corresponds to a modulation of the conducting area of a cluster. This region is usually not connected. However, Swillens et al. showed that the spatial arrangement of $\mathrm{IP}_{3} \mathrm{R}$ channels does not influence the $\mathrm{Ca}^{2+}$ dynamics at an open cluster [30]. Therefore, we map the area of all conducting release channels to an area of the same size concentric to the cluster area. Let $a$ denote the radius of this region, $N$ the total number of channels per cluster and $n_{o}$ the number of open channels, then $a=a_{0} \sqrt[3]{n_{o} / N}$. That reflects the above notion that the volume of the conducting sphere corresponds to the volume that is occupied by the fraction $n_{o} / N$ of open channels. If $n_{o}=0$ then $a=0$, and $a$ takes the maximal value $a_{0}$ if all $N$ channels are open.

The deterministic dynamics of this cluster model has been investigated in $[32,33]$. In addition to $\mathrm{IP}_{3}$ mediated $\mathrm{Ca}^{2+}$ liberation, we considered sarcoendoplasmic reticulum calcium ATPase (SERCA) pumps, which transport $\mathrm{Ca}^{2+}$ from the cytosol to the ER, and a leak flux. The stationary $\mathrm{Ca}^{2+}$ concentration profile that results from these three fluxes is

$$
c(r)=\left[A(a) \frac{\sinh \left(k_{1} r\right)}{r}+e_{1}\right] \Theta(a-r)+\left[B(a) \frac{\exp \left(-k_{2} r\right)}{r}+e_{2}\right] \Theta(r-a),
$$

where

$$
\begin{aligned}
A(a) & =\frac{l\left(k_{2} a+1\right)}{\cosh \left(k_{1} a\right) k_{1}+\sinh \left(k_{1} a\right) k_{2}}, \\
B(a) & =\frac{l\left(\sinh \left(k_{1} a\right)-\cosh \left(k_{1} a\right) k_{1} a\right)}{\exp \left(-k_{2} a\right)\left(\cosh \left(k_{1} a\right) k_{1}+\sinh \left(k_{1} a\right) k_{2}\right)},
\end{aligned}
$$


with

$$
l=\frac{-k_{c} k_{p} E}{\left(k_{l}+k_{p}+k_{c}\right)\left(k_{l}+k_{p}\right)}, \quad k_{1}=\sqrt{\frac{k_{l}+k_{p}+k_{c}}{D}}, \quad k_{2}=\sqrt{\frac{k_{l}+k_{p}}{D}},
$$

and $e_{1}:=\left(k_{l}+k_{c}\right) E /\left(k_{l}+k_{p}+k_{c}\right), e_{2}:=k_{l} E /\left(k_{l}+k_{p}\right)$. The constants $k_{l}$, $k_{p}$ and $k_{c}$ denote the leak flux coefficient, the strength of the SERCA pumps and the channel flux coefficient, respectively. The diffusion coefficient is given by $D$. $E$ denotes the concentration of free $\mathrm{Ca}^{2+}$ in the ER.

Simulations have demonstrated that the $\mathrm{Ca}^{2+}$ concentration rapidly equilibrates upon a change in the number of open channels [31]. Hence, we will approximate the $\mathrm{Ca}^{2+}$ dynamics by its stationary value in the remainder of this work. The number of open channels $n_{o}$ uniquely determines the $\mathrm{Ca}^{2+}$ concentration according to equation (1) and $a=a_{0} \sqrt[3]{n_{o} / N}$. The focus of the two subsequent sections is the calculation of $n_{o}$.

\section{Master equation}

The number of open channels $n_{o}$ depends on the state of the subunits of the $\mathrm{IP}_{3} \mathrm{Rs}$. A state of a subunit is determined by the occupation of its binding sites. The De Young Keizer (DK) model has three binding sites per subunit and hence eight subunit states. We reduce these eight states in two steps to three states. Firstly, we eliminate the $\mathrm{IP}_{3}$ dynamics adiabatically since $\mathrm{IP}_{3}$ binding and unbinding are much faster than the $\mathrm{Ca}^{2+}$ dynamics in the framework of this model. The resulting four states are labeled by a binary pair $i j$, where the first index represents the $\mathrm{Ca}^{2+}$ activating binding site and the second the $\mathrm{Ca}^{2+}$ inhibiting binding site [6]. An index equals 1 when the binding site is occupied and 0 otherwise, e.g. 10 corresponds to the activatable state of a subunit. The second approximation uses the fact that we are interested in activation starting from a stationary state. Transitions among the inhibited states 11 and 01 have little impact on that activation process. Moreover, these states are rarely populated during puff initiation. Consequently we lump the two inhibited state into one state $\bar{h}$. Figure 1 depicts the transition scheme for this 3 state model. The transition rates follow from [5] and [6].

Modeling the dynamics of an $\mathrm{IP}_{3} \mathrm{R}$ on the basis of its subunits leads to various consequences for a cluster of $N \mathrm{IP}_{3} \mathrm{Rs}$. As long as every $\mathrm{IP}_{3} \mathrm{R}$ is treated individually and subunits are assigned to individual channels - as has been done in stochastic simulations [6] - the state of the cluster is uniquely determined by the states of its subunits. However, an approach based on a population of subunits not grouped into individual channels is more suitable for the derivation of master equations and Fokker-Planck equations which we would like to use. That requires to determine the number of open channels from the total number of activatable subunits in the subunit population. We assume that the activatable subunits are randomly scattered across the channels. The distribution of the $n_{10}$ activatable subunits on the $4 N$ subunits of 


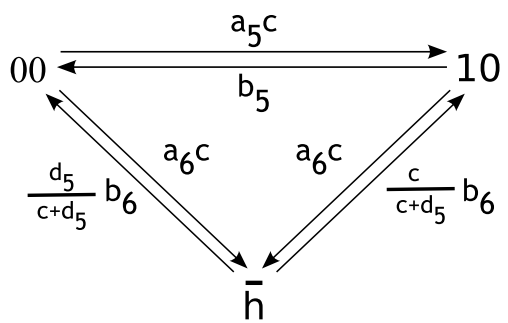

Fig. 1. Transition scheme for the three state model of the $\mathrm{IP}_{3}$ receptor. $d_{5}=b_{5} / a_{5}$ is the dissociation constant for $\mathrm{Ca}^{2+}$ activation, $b_{6}$ the dissociation rate of $\mathrm{Ca}^{2+}$ from the inhibiting site averaged over both $\mathrm{IP}_{3}$ binding states. We denote the number of subunits in one of the three states by $n_{10}, n_{00}$ and $n_{\bar{h}}$

a cluster decides upon the value of $n_{o}$ and hence the $\mathrm{Ca}^{2+}$ concentration. We show in the appendix that this distribution is sharply peaked around its mean value. Therefore, we set $n_{o}=\left\langle n_{o}\right\rangle=n_{a} . n_{a}$ is defined in equation (52).

The stochastic nature of $\mathrm{Ca}^{2+}$ release through $\mathrm{IP}_{3} \mathrm{Rs}$ entails that the exact number of subunits in either of the three states 10,00 or $\bar{h}$ at a given time $t$, i.e. the triplet $\left(n_{10}(t), n_{\bar{h}}(t), n_{00}(t)\right)$, cannot be specified exactly any more. On the contrary, only the probability $P\left(n_{10}, n_{\bar{h}}, n_{00} ; t\right)$ to find a certain realization of $\left(n_{10}, n_{\bar{h}}, n_{00}\right)$ at time $t$ is accessible. Since the total number of subunits is fixed, the values of $n_{10}$ and $n_{\bar{h}}$ suffice to specify the triplet $\left(n_{10}, n_{\bar{h}}, n_{00} ; t\right)$, so that $P\left(n_{10}, n_{\bar{h}}, n_{00} ; t\right)=P\left(n_{10}, n_{\bar{h}} ; t\right)$.

The probability $P\left(n_{10}, n_{\bar{h}} ; t\right)$ changes in the time interval $[t, t+d t]$ due to two opposing processes: Being in $\left(n_{10}, n_{\bar{h}}\right)$ at time $t$, binding or unbinding of $\mathrm{Ca}^{2+}$ alters $n_{10}$ or $n_{\bar{h}}$ during $d t$ and hence reduces $P\left(n_{10}, n_{\bar{h}} ; t\right)$. On the other hand, transition from states as $\left(n_{10}+1, n_{\bar{h}}\right)$ or $\left(n_{10}-1, n_{\bar{h}}\right)$ into $\left(n_{10}, n_{\bar{h}}\right)$ increases $P\left(n_{10}, n_{\bar{h}} ; t\right)$. Taking all possible reactions according to the figure 1 into account, the time evolution of $P\left(n_{10}, n_{\bar{h}} ; t\right)$ is captured by the master equation [34]

$$
\begin{aligned}
& \dot{P}\left(n_{10}, n_{\bar{h}} ; t\right)= \\
& -\left[n_{10}\left[b_{5}+a_{6} c\left(n_{10}\right)\right]+n_{\bar{h}} b_{6}\right] P\left(n_{10}, n_{\bar{h}} ; t\right)+\left[n_{10}+1\right] b_{5} P\left(n_{10}+1, n_{\bar{h}} ; t\right) \\
& -n_{\bar{h}} b_{6} P\left(n_{10}, n_{\bar{h}} ; t\right)+\left[h N-n_{\bar{h}}-n_{10}+1\right] a_{6} c\left(n_{10}\right) P\left(n_{10}, n_{\bar{h}}-1 ; t\right) \\
& -\left[h N-n_{10}-n_{\bar{h}}\right]\left[a_{6} c\left(n_{10}\right)+a_{5} c\left(n_{10}\right)\right] P\left(n_{10}, n_{\bar{h}} ; t\right) \\
& +\frac{b_{6} c\left(n_{10}-1\right)\left[n_{\bar{h}}+1\right]}{c\left(n_{10}-1\right)+d_{5}} P\left(n_{10}-1, n_{\bar{h}}+1 ; t\right)+\frac{b_{6} d_{5}\left[n_{\bar{h}}+1\right]}{c\left(n_{10}\right)+d_{5}} P\left(n_{10}, n_{\bar{h}}+1 ; t\right) \\
& +\left[h N-n_{\bar{h}}-n_{10}+1\right] a_{5} c\left(n_{10}-1\right) P\left(n_{10}-1, n_{\bar{h}} ; t\right) \\
& +\left[n_{10}+1\right] a_{6} c\left(n_{10}+1\right) P\left(n_{10}+1, n_{\bar{h}}-1 ; t\right) .
\end{aligned}
$$

For instance being in $\left(n_{10}, n_{\bar{h}}\right)$, the term proportional to $a_{6} c$ in the first line denotes a transition from 10 to $\bar{h}$, so that the final state is $\left(n_{10}-1, n_{\bar{h}}+1\right)$. The $\mathrm{Ca}^{2+}$ concentration is given by equation (1). The $\mathrm{Ca}^{2+}$ concentration 
in the master equation depends on $n_{10}$, which is indicated by the notation $c\left(n_{10}\right)$. The radius $a$ in equation (1) follows from the number of activatable subunits as $a=a_{0} \sqrt[3]{n_{a} / N}$ according to the preceding discussions.

The adiabatic elimination of the $\mathrm{IP}_{3}$ dynamics leads to non-integer values for the number of open channels. That demands a careful interpretation of the size of the conducting membrane patch, which was assumed to take only discrete values due to the discreteness of $n_{o}$. One approach is to truncate the rational values of $n_{o}$ as $\left[n_{o}\right]_{+}$, where $\left[n_{o}\right]_{+}$denotes the largest integer that is less or equal $n_{o}$. It entails $c=c_{b}$ as long as $n_{0}<1$, where $c_{b}$ denotes the base level of the $\mathrm{Ca}^{2+}$ concentration. This approach favors the closed configuration during puff initiation. In another approach we will keep the non-integer value of $n_{o}$ and consider $a$ as a quasi continuous function. We will discuss the effects of both approaches with respect to puff initiation.

Equation (4) is an accurate description of the stochastic dynamics represented by the scheme in Figure 1. We will derive approximations like FokkerPlanck equations to calculate escape time characteristics from this master equation.

\section{Fokker-Planck equations}

The discrete nature of master equations often impedes an analytic treatment. That holds in particular for master equations with nonlinearities or artificial boundary conditions. In these cases, several approximations have been put forward [34, 25, 18, 11, 12]. Despite the plethora of methods, there is still no consensus which approximation is best [9]. Each of them possesses advantages and drawbacks, so that the problem at hand finally decides which procedure to use. We will concentrate on van Kampen's $\Omega$ expansion and a method that is similar to a Kramers-Moyal expansion. The latter keeps the nonlinearities of the master equation in the fluctuations, whereas the former approximates them in a linear fashion. Moreover, van Kampens's expansion is only valid when the macroscopic equation displays a single stable fixed point.

The $\Omega$ expansion requires a small parameter $1 / \Omega$ in the master equation, which for our purposes is the inverse number of subunits, i.e. $\Omega=4 N$. The systematic expansion of equation (4) in powers of $\Omega$ is based on the transformations $n_{10}=\Omega \phi(t)+\Omega^{1 / 2} \xi$ and $n_{\bar{h}}=\Omega \psi(t)+\Omega^{1 / 2} \eta$. They decompose the variables of the master equation into macroscopic parts $(\phi, \psi)$ and fluctuations $(\xi, \eta)$. Inserting this ansatz into equation (4), the first non vanishing order of $\Omega$ yields the macroscopic equations

$$
\begin{aligned}
& \frac{\partial \phi}{\partial t}=-\phi\left(a_{5} c+a_{6} c+b_{5}\right)+\psi\left(\frac{b_{6} c}{c+d_{5}}-a_{5} c\right)+a_{5} c, \\
& \frac{\partial \psi}{\partial t}=-\left(a_{6} c+b_{6}\right) \psi+a_{6} c
\end{aligned}
$$


with $c=c\left(a_{0} \sqrt[3]{\phi_{a}}\right)$ and $\phi_{a}:=r^{3} \phi^{3}(4-3 r \phi) . r$ denotes the fraction of subunits in the state 10 that are activated: $r:=I /\left(I+d_{1}\right)$. Equations (5) correspond to the rate equations that are associated with the transition scheme in figure 1 , when the conservation condition $n_{10}+n_{\bar{h}}+n_{00}=4 N$ is applied. Note that $\phi_{a}$ is the continuous limit $(N \rightarrow \infty)$ of equation (52). Therefore, $\phi_{a}$ is the probability that at least 3 of the 4 subunits of an $\mathrm{IP}_{3} \mathrm{R}$ are activated. The solutions of equation (5) represent the deterministic part of the above transformation of variables. They have the stationary values

$$
\bar{\phi}=\frac{d_{6} c}{\left(c+d_{5}\right)\left(c+d_{6}\right)}, \quad \bar{\psi}=\frac{c}{c+d_{6}},
$$

which agree with results in [33]. $d_{6}=d_{2}\left(I+d_{1}\right) /\left(I+d_{3}\right)$ is an effective dissociation constant. $d_{2}$ denotes the dissociation constant for $\mathrm{Ca}^{2+}$ inhibition when the $\mathrm{IP}_{3}$ binding site is ligated, $d_{1}$ and $d_{3}$ represent the dissociation constants of $\mathrm{IP}_{3}$ binding [5].

The next order in $\Omega$ determines the fluctuations through the probability $P\left(n_{10}, n_{\bar{h}} ; t\right)$

$=P\left(\Omega \phi+\Omega^{1 / 2} \xi, \Omega \psi+\Omega^{1 / 2} \eta ; t\right)=: \Pi(\xi, \eta ; t)$ according to

$$
\begin{aligned}
\frac{\partial \Pi}{\partial t}= & -\left[g_{11} \frac{\partial}{\partial \xi}+g_{21} \frac{\partial}{\partial \eta}\right](\xi \Pi)-\left[g_{12} \frac{\partial}{\partial \xi}+g_{22} \frac{\partial}{\partial \eta}\right](\eta \Pi) \\
& +\frac{1}{2}\left[h_{11} \frac{\partial^{2}}{\partial \xi^{2}}+2 h_{12} \frac{\partial^{2}}{\partial \eta \partial \xi}+h_{22} \frac{\partial^{2}}{\partial \eta^{2}}\right] \Pi .
\end{aligned}
$$

The matrices $\left(g_{i j}\right)$ and $\left(h_{i j}\right)$ with $h_{12}=h_{21}$ are defined as

$$
\begin{aligned}
g_{11}:=b_{6} d_{5} \psi c^{1} /\left(c+d_{5}\right)^{2}-a_{6}\left(c+\phi c^{1}\right)-b_{5}-a_{5}\left(c-(1-\phi-\psi) c^{1}\right), \\
g_{21}:=a_{6} c^{1}-a_{6} \psi c^{1} \\
g_{12}:=b_{6} c /\left(c+d_{5}\right)-a_{5} c \\
g_{22}:=-\left(a_{6} c+b_{6}\right)
\end{aligned}
$$

and

$$
\begin{aligned}
& h_{11}:=a_{5}(1-\psi-\phi) c+b_{6} \psi c /\left(c+d_{5}\right)+a_{6} \phi c+b_{5} \phi, \\
& h_{21}:=-b_{6} \psi c /\left(c+d_{5}\right)-a_{6} \phi c, \\
& h_{22}:=a_{6}(1-\psi) c+b_{6} \psi .
\end{aligned}
$$

with

$$
c^{1}:=\frac{d c}{d a}\left(a_{0} \sqrt[3]{\phi_{a}}\right) \frac{a_{0}}{3} \sqrt[3]{\phi_{a}^{-2}}\left[18 r^{4} \phi^{3}-12 r^{3} \phi^{3}-12 r^{3} \phi^{2}\right] .
$$

Equation (10) arises from inserting equation (52) into $a=a_{o} \sqrt[3]{n_{a} / N}$ and then expanding $c(a)$ in powers of $\Omega$. The matrix $\left(g_{i j}\right)$ coincides with the matrix of the linearized macroscopic equations (5). The fluctuations enter through the matrix $\left(h_{i j}\right)$. The Hurwitz criterion [15] assures that this matrix is positive 
semi definite, which means that equation (7) is a linear multivariate FokkerPlanck equation.

The linear treatment of the noise in equation (7) has cast some doubt on the validity of the $\Omega$ expansion. Therefore, a different class of Fokker-Planck equations have been proposed that keep the nonlinearities of the master equation. Kramers and Moyal have treated the shifts $n_{10} \pm 1, n_{\bar{h}} \pm 1$ of $n_{10}$ and $n_{\bar{h}}$ in equation (4) by means of a Taylor expansion [18, 25]. Following this procedure and defining the new variables $\phi:=n_{10} / \Omega$ and $\psi:=n_{\bar{h}} / \Omega$, we obtain a Fokker-Planck equation for the probability $p=p(\phi, \psi, t)$ :

$$
\begin{aligned}
\frac{\partial p}{\partial t}= & \frac{\partial}{\partial \phi}\left[\phi a_{6} c+\phi b_{5}-(1-\psi-\phi) a_{5} c-\frac{b_{6} c}{c+d_{5}} \psi\right] p \\
& +\frac{\partial^{2}}{2 \Omega \partial \phi^{2}}\left[\phi a_{6} c+\phi b_{5}+(1-\psi-\phi) a_{5} c+\frac{b_{6} c}{c+d_{5}} \psi\right] p \\
& +\frac{\partial}{\partial \psi}\left[b_{6}-(1-\psi) a_{6} c\right] p+\frac{\partial^{2}}{2 \Omega \partial \psi^{2}}\left[b_{6}+(1-\psi) a_{6} c\right] p \\
& -\frac{\partial}{\Omega \partial \psi \partial \phi}\left[\phi a_{6} c+\frac{b_{6} c}{c+d_{5}}\right] p .
\end{aligned}
$$

The nonlinearities are introduced through $c=c\left(a_{0} \sqrt[3]{\phi_{a}}\right)$ with $\phi_{a}$ defined as after equation (5).

Equations (4), (7) and (11) constitute the starting point for a systematic study of puff frequencies. Given a configuration $\left(n_{10}^{0}, n_{\bar{h}}^{0}\right)$ at time $t=0$, they all yield the probability for a configuration $\left(n_{10}^{t}, n \frac{t}{h}\right)$ at time $t>0$. If we identify $\left(n_{10}^{0}, n_{\bar{h}}^{0}\right)$ with the resting state of a cluster and $\left(n_{10}^{t}, n_{\bar{h}}^{t}\right)$ with the first channel opening, such a transition in the configuration space gives the probability for a $\mathrm{Ca}^{2+}$ puff. Consequently, we interpret a puff as an escape process from the state $\left(n_{10}^{0}, n_{\bar{h}}^{0}\right)$ to the state $\left(n_{10}^{t}, n_{\bar{h}}^{t}\right)$. Although the above equations allow the calculation of this escape probability, no general solutions are known for two dimensional escape processes (see [13] for a recent result). However, the time scale separation between $\mathrm{Ca}^{2+}$ activation and $\mathrm{Ca}^{2+}$ inhibition leads to a reduction of the two dimensional equations to one dimension. Since the inhibiting processes are much slower than binding and unbinding of $\mathrm{Ca}^{2+}$ to the activating binding site, we assume that $n_{\bar{h}}$ remains unchanged during the initiation of a puff. That is identical to setting $n_{\bar{h}}=$ const, and the master equation simplifies to

$$
\begin{aligned}
\dot{P}\left(n_{10}\right)= & -\frac{b_{6} c\left(n_{10}\right)}{c\left(n_{10}\right)+d_{5}} n_{\bar{h}} P\left(n_{10}\right)+\frac{b_{6} c\left(n_{10}-1\right)}{c\left(n_{10}-1\right)+d_{5}} n_{\bar{h}} P\left(n_{10}-1\right) \\
& -a_{6} c\left(n_{10}\right) n_{10} P\left(n_{10}\right)+a_{6}\left(n_{10}+1\right) c\left(n_{10}+1\right) P\left(n_{10}+1\right) \\
& -\left(4 N-n_{10}-n_{\bar{h}}\right) a_{5} c\left(n_{10}\right) P\left(n_{10}\right)-b_{5} n_{10} P\left(n_{10}\right) \\
& +\left(4 N-n_{10}-n_{\bar{h}}+1\right) a_{5} c\left(n_{10}-1\right) P\left(n_{10}-1\right) \\
& +b_{5}\left(n_{10}+1\right) P\left(n_{10}+1\right) .
\end{aligned}
$$

For a later analysis, it is convenient to rewrite equation (12) in the form 


$$
\dot{P}\left(n_{10}\right)=-\left(g_{n_{10}}+r_{n_{10}}\right) P\left(n_{10}\right)+g_{n_{10}-1} P\left(n_{10}-1\right)+r_{n_{10}+1} P\left(n_{10}+1\right)
$$

with

$$
\begin{aligned}
& g_{n_{10}}=\frac{b_{6} c\left(n_{10}\right)}{c\left(n_{10}\right)+d_{5}}[\Omega \bar{\psi}]+\left(4 N-[\Omega \bar{\psi}]-n_{10}\right) a_{5} c, \\
& r_{n_{10}}=b_{5} n_{10}+a_{6} c\left(n_{10}\right) n_{10},
\end{aligned}
$$

and $\bar{\psi}$ defined as in equation (6). From equation (12), we could again derive Fokker-Planck equations in the same manner as before. However, a more direct approach for the one dimensional $\Omega$ expansion is setting $\eta$ equal to zero in equation (7) due to $n_{\bar{h}}=$ const. Keeping only the derivatives with respect to $\phi$ in equation (11) gives the nonlinear Fokker-Planck equation. Note that these one dimensional equations are only valid during the initiation phase of a puff, whereas equations (4), (7) and (11) capture the full time evolution. Nevertheless, we will concentrate on equation (12) and the entailing FokkerPlanck equations in the remainder of this work, because they admit analytic solutions and provide far reaching insights into puff frequencies. The existence of analytic solutions is one of the most prominent features of van Kampen's expansion, so that we will treat the corresponding Fokker-Planck equation most generally in the next section.

\section{Escape times}

The initiation of a $\mathrm{Ca}^{2+}$ puff corresponds to an escape from the stationary state to the first channel opening. That requires the definition of the boundaries of the phase space area from which the escape occurs. Since we restrict the discussion to one dimension in phase space, the boundary consists of two points. We see from equation (12) that the lower boundary $d$ is at $n_{10}=0$ and that it is reflecting. That agrees with the interpretation of $n_{10}$ as the number of activatable subunits, which is always positive. The value of the upper boundary $b$ is chosen such that the number of open channels $n_{o}=1$. The upper boundary corresponds to the escape site, so that the boundary condition is of absorbing type [8].

The time $t$ to reach the absorbing boundary is a stochastic variable. It is described by the probability density $\rho(t)$ i.e. $\rho(t) d t$ is the probability that the stochastic process reaches $b$ between $t$ and $t+d t$. $\rho$ is most conveniently computed from $G(x, t)=1-\int_{0}^{t} \rho(x, \tau) d \tau$, which represents the probability that $d \leq n_{10}<b$ at time $t$ when it started at $x=n_{10}^{0}$ at $t=0$. The time evolution of $G$ is governed by $\tilde{\mathcal{L}}$, which is the adjoint of the FokkerPlanck operator $\mathcal{L}[8]$. Up to now, no general solution has been obtained for arbitrary $\mathcal{L}$. Yet, an analytic expression exists for $G$ in the case of a linear Fokker-Planck operator, e.g. van Kampen's $\Omega$ expansion. Since the following derivation always holds and is not restricted to the current problem, 
we introduce new constants $v$ and $w$. They are given by $v=-g_{11}$ and $w=h_{11}$ defined as in equations (8a) and (9a), respectively, in the present study. $G$ obeys the linear backward Fokker-Planck equation [8]:

$$
\frac{\partial G(x, t)}{\partial t}=-v x \frac{\partial G(x, t)}{\partial x}+\frac{w}{2} \frac{\partial^{2} G(x, t)}{\partial x^{2}}, \quad v, w>0,
$$

with initial and boundary conditions

$$
G(x, 0)=\left\{\begin{array}{ll}
1, & d \leq x \leq b \\
0, & \text { else }
\end{array}, \quad \frac{\partial G(d, t)}{\partial x}=0 \quad \forall t, \quad G(b, t)=0 \quad \forall t .\right.
$$

The initial condition states that $d \leq x<b$ at $t=0$ with probability one. The reflecting boundary condition at $x=d$ in the adjoint Fokker-Planck equation is expressed by a no-flux boundary condition. Setting $G \equiv 0$ at the right boundary corresponds to an absorbing boundary. We solve equation (15) with the ansatz $G(x, t)=\exp (-\lambda t) u(x), \lambda \geq 0$ so that it reduces to the ordinary differential equation

$$
\frac{d^{2} u}{d x^{2}}-\frac{2 v x}{w} \frac{d u}{d x}+\frac{2 \lambda}{w} u=0 .
$$

Applying the transformation $z:=x^{2} / 4$ we find for $\bar{u}(z):=u(x)$

$$
z \frac{d^{2} \bar{u}}{d z^{2}}+\left(\frac{1}{2}-\frac{4 v z}{w}\right) \frac{d \bar{u}}{d z}+\frac{2 \lambda}{w} \bar{u}=0 .
$$

It equals Kummer's equation for $\tilde{u}(\tilde{z}):=\bar{u}(z)$ with $\tilde{z}:=4 v z / w$

$$
\tilde{z} \frac{d^{2} \tilde{u}}{d \tilde{z}^{2}}+\left(\frac{1}{2}-\tilde{z}\right) \frac{d \tilde{u}}{d \tilde{z}}+\frac{\lambda}{2 v} \tilde{u}=0 .
$$

Two independent solutions of equation (17) are [1]

$$
u_{1}(x):=M\left(-\frac{\lambda}{2 v}, \frac{1}{2}, \frac{v x^{2}}{w}\right), \quad u_{2}(x):=x M\left(\frac{1}{2}-\frac{\lambda}{2 v}, \frac{3}{2}, \frac{v x^{2}}{w}\right) .
$$

$M$ designates the confluent hypergeometric function

$$
M(a, b, x):=\sum_{k=0}^{\infty} \frac{(a)_{k}}{(b)_{k}} \frac{x^{k}}{k !}
$$

where $(a)_{0}:=1$ and $(a)_{k}:=a(a+1) \ldots(a+k-1)$. The boundary condition at $n_{10}=b$ entails that a solution of equation (17) is

$$
v(x):=C_{1}\left[u_{1}(x)-\frac{u_{1}(b)}{u_{2}(b)} u_{2}(x)\right]=u_{1}(x)-\frac{u_{1}(b)}{u_{2}(b)} u_{2}(x) .
$$


Without loss of generality we set $C_{1}=1$ because it merely serves as normalization. The second boundary condition fixes the still unknown eigenvalues $\lambda$. They constitute an infinite countable set $\left\{\lambda_{n}\right\}$ due to the finiteness of $d$ and $b$. Therefore, the general solution of equation (15) can be expressed as

$$
G(x, t)=\sum_{n=0}^{\infty} a_{n} \exp \left(-\lambda_{n} t\right) v_{n}(x)
$$

The subscript of $v_{n}(x)$ indicates that equation (22) has to be evaluated at $\lambda=\lambda_{n}$ (see equation (20)). The coefficients $a_{n}$ are determined by the initial condition $G(x, 0)$ which results in

$$
a_{n}=\int_{d}^{b} r(x) v_{n}(x) d x / \int_{d}^{b} r(x) v_{n}^{2}(x) d x, \quad r(x):=\frac{2}{w} \exp \left(-\frac{v}{w} x^{2}\right) .
$$

Here we used the orthogonality relation of the eigenfunctions $v_{n}(x)$ :

$$
\int_{d}^{b} v_{n}(x) v_{m}(x) r(x) d x=\delta_{m, n} \int_{d}^{b} v_{n}^{2}(x) r(x) d x .
$$

The probability $\rho(x, t)$ that the absorbing state is reached between $t$ and $t+d t$ is readily computed from $G(x, t)$ as $\rho=-\partial_{t} G(x, t)$. Note that $\rho$ is already normalized due to the initial condition $G(x, 0)$. Hence, the mean first passage time $T(x)$ equals

$$
T(x):=\langle t(x)\rangle=\int_{0}^{\infty} t \rho(x, t) d t=-\int_{0}^{\infty} t \partial_{t} G(x, t) d t=\sum_{n} \frac{a_{n} v_{n}(x)}{\lambda_{n}} .
$$

Equation (26) includes an infinite number of eigenvalues. We found that the first three terms of the sum over $n$ were sufficient to achieve results indistinguishable from the exact results of equation (30).

An alternative approach to the mean first passage time follows from the differential equation [8]

$$
-v x \frac{d T(x)}{d x}+\frac{w}{2} \frac{d^{2} T(x)}{d x^{2}}=-1,
$$

with the solution

$$
T(x)=\frac{2}{w} \int_{x}^{b} \frac{d y}{h(y)} \int_{d}^{y} h(z) d z, \quad h(x):=\exp \left\{-\frac{v}{w}\left(x^{2}-d^{2}\right)\right\} .
$$

Performing the $z$ integration we find 


$$
\begin{aligned}
T(x)= & \sqrt{\frac{\pi}{v w}} \int_{x}^{b} d y \exp \left(\frac{v}{w} y^{2}\right) \operatorname{erf}\left(\sqrt{\frac{v}{w}} y\right) \\
& +\frac{\pi}{2 v} \operatorname{erf}\left(\sqrt{\frac{v}{w}} d\right)\left\{\operatorname{erfi}\left(\sqrt{\frac{v}{w}} x\right)-\operatorname{erfi}\left(\sqrt{\frac{v}{w}} b\right)\right\} .
\end{aligned}
$$

The functions $\operatorname{erf}(x)$ and $\operatorname{erfi}(x)=\operatorname{erf}(i x) / i$ denote the Gaussian error function and the imaginary Gaussian error function, respectively. The remaining integral can be solved by series expansion so that the final expression for the mean first passage time takes the form

$$
\begin{aligned}
T(x)= & \frac{b^{2}}{w} F_{2 ; 2}\left(1,1 ; \frac{3}{2}, 2 ; \frac{w}{v} b^{2}\right)-\frac{x^{2}}{w} F_{2 ; 2}\left(1,1 ; \frac{3}{2}, 2 ; \frac{w}{v} x^{2}\right) \\
& +\frac{\pi}{2 v} \operatorname{erf}\left(\sqrt{\frac{v}{w}} d\right)\left\{\operatorname{erfi}\left(\sqrt{\frac{v}{w}} x\right)-\operatorname{erfi}\left(\sqrt{\frac{v}{w}} b\right)\right\} .
\end{aligned}
$$

We employed the generalized hypergeometric function

$$
F_{p ; q}\left(a_{1}, \ldots, a_{p} ; b_{1}, \ldots, b_{q} ; x\right)=\sum_{l=0}^{\infty} \frac{\left(a_{1}\right)_{l} \cdots\left(a_{p}\right)_{l}}{\left(b_{1}\right)_{l} \cdots\left(b_{q}\right)_{l}} \frac{x^{l}}{l !},
$$

and used the identiy

$$
\frac{j !}{2 j+2} \sum_{l=0}^{j} \frac{(-1)^{l}}{(2 l+1)(j-l) ! l !}=\frac{1}{2} \frac{(1)_{j}(1)_{j}}{\left(\frac{3}{2}\right)_{j}(2)_{j}} .
$$

We defer the proof to the appendix. The reason for presenting two methods for evaluating the mean first passage time is based on their different scopes of applicability. If we were only interested in $T$, then equation (30) would be preferable because it requires less computation. However, we are limited to the first moment [8]. The advantage of the first approach is that we obtain any moment by one integration. Moreover, we have access to the time evolution of the escape process which allows for a more detailed analysis.

The above results could only be obtained analytically because the corresponding Fokker-Planck equation was linear. In the case of a nonlinear FokkerPlanck equation, all quantities have to be computed numerically. The mean first passage time is evaluated best from a generalization of equation (28). For $\mathcal{L}=-\partial_{x} A(x)+\partial_{x}^{2} B(x) / 2$ we find $[8]$

$$
T(x)=2 \int_{x}^{b} \frac{d y}{h(y)} \int_{d}^{y} \frac{h(z)}{B(z)} d z, \quad h(x):=\exp \left\{\int_{d}^{x} \frac{2 A(y)}{B(y)} d y\right\} .
$$

The study of Fokker-Planck equations instead of master equations is often motivated by easier treatment. That holds in particular in higher dimensions, 
because a broader spectrum of tools is available for Fokker-Planck equations than for master equations [27] and even analytical calculations may be possible as in the case of equation (12). That constitutes one of the reasons for the derivations in section 4. However, Fokker-Planck equations always represent approximations. The only way to test their quality is a comparison with results obtained from a master equation.

To this end, we consider a general one step process, to which class equation (12) belongs. We assume that this jump process starts at a site $m$ at $t=0$. Being at site $n$ the particle hops to the right with a rate $g_{n}$ and to the left with a rate $r_{n}$, respectively. When it reaches the left boundary $L$, it is reflected. Then, the mean first passage time to arrive at a site $R>m$ reads [34]

$$
T_{R, m}=\sum_{i=m}^{R-1}\left(\frac{1}{g_{i}}+\sum_{l=L+1}^{i} \frac{r_{i} r_{i-1} \cdots r_{l}}{g_{i} g_{i-1} \cdots g_{l}} \frac{1}{g_{l-1}}\right) .
$$

That allows us to estimate the validity of the preceding approximations. The transition rates $r_{i}$ and $g_{i}$ follow from equation (14) for the current investigation.

\section{Results}

\subsection{Mean first passage time}

The calculation of the mean first passage times according to equations (26), (33) and (34) necessitates a further specification of the boundaries. Since we consider a cluster with $N$ channels, the upper boundary $\phi_{b}$ for the nonlinear Fokker-Planck equation is given by the solution of $\left(r \phi_{b}\right)^{3}\left(4-3 r \phi_{b}\right)=1 / N$. The left hand side corresponds to the fraction of open channels as discussed after equation (5). For the lower boundary, we have $\phi=0$. This value holds for the master equation, too. The upper boundary for the master equation is obtained by rounding off $\Omega \phi_{b}$ to its nearest integer value $\left[\Omega \phi_{b}\right]$. Before specifying the boundary conditions for van Kampen's expansion we note that it describes the strength of the fluctuations $\xi$ around the fixed point $\bar{\phi}$. The left boundary is imposed by $n_{10}>0$, whereas the right boundary has to satisfy $\phi_{b}=\bar{\phi}+\Omega^{-1 / 2} \xi$. Consequently, the boundaries of $\xi$ are $-\Omega^{1 / 2} \bar{\phi}$ and $\left(\phi_{b}-\bar{\phi}\right) \Omega^{1 / 2}$, respectively, with $\bar{\phi}$ given by equation (6).

The mean first passage time depends strongly on the $\mathrm{Ca}^{2+}$ concentration (see e.g. equations (14) and (34)). The results presented throughout the sections $6.1-6.3$ are calculated with a constant base level concentration. The number of open channels is an integer variable and there is no $\mathrm{Ca}^{2+}$ channel flux before the first channel opens. The $\mathrm{Ca}^{2+}$ concentration remains at a steady value until a $\mathrm{Ca}^{2+}$ puff occurs. That leads to $c^{1} \equiv 0$ in equation (10) and to coefficients linear in $\phi$ and $\psi$ in equation (11). 

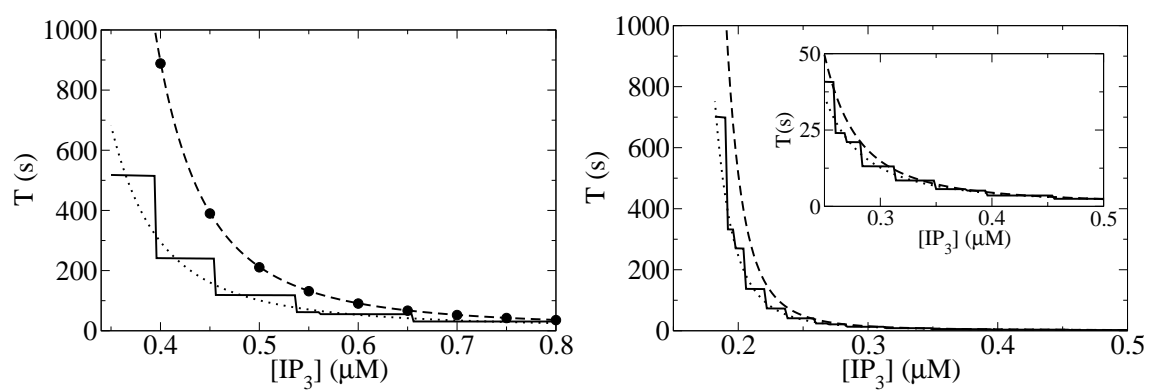

Fig. 2. Mean first passage time for $c_{b}=50 n \mathrm{M}$ (left) and $c_{b}=80 n \mathrm{M}$ (right) computed from the master equation (solid), the $\Omega$ expansion (dashed) and the nonlinear Fokker-Planck equation (dotted) for $d_{1}=0.13 \mu \mathrm{M}, d_{2}=3 \mu \mathrm{M}, d_{3}=0.9434 \mu \mathrm{M}, d_{4}=$ $0.4133 \mu \mathrm{M}, d_{5}=0.24 \mu \mathrm{M}, a_{2}=a_{4}=0.2(\mu \mathrm{Ms})^{-1}, a_{5}=5(\mu \mathrm{Ms})^{-1}, N=25$. The dots in the left panel represent the variance of the $\Omega$ expansion. The inset in the right panel shows a blow up of the plot for large $\mathrm{IP}_{3}$ concentration.

Figure 2 depicts the mean first passage time as a function of the $\mathrm{IP}_{3}$ concentration for two different values of the basal $\mathrm{Ca}^{2+}$ concentration.

The master equation and the two Fokker-Planck equations exhibit an increase of the mean first passage time with decreasing $\mathrm{IP}_{3}$ concentration. This increment diverges for lower values of the $\mathrm{IP}_{3}$ concentration.

The nonlinear Fokker-Planck equation interpolates the master equation very efficiently. The results agree well with experimental findings for puff periods, although the mean first passage time only constitutes its mean stochastic fraction [21]. The discreteness of the master equation leads to discontinuities in the mean first passage time. The plateaus correspond to ranges of $\phi_{b}$ that are mapped to a single integer for the absorbing boundary of the master equation. Whenever that integer increases by 1 , a jump occurs in the mean first passage time. Van Kampen's expansion yields good results for higher $\mathrm{IP}_{3}$ concentrations, but overestimates the escape times otherwise (figure 2).

Figure 3 depicts the influence of the base level on the mean first passage time. The higher the basal concentration in this regime, the faster the first channel opens. Van Kampen's expansion improves with increasing base level as a comparison between the two panels in figure 2 and the right panel of figure 3 shows. The zigzag behavior of the relative difference $\tau:=\left(T_{v K}-\right.$ $\left.T_{M E}\right) / T_{M E}$ results from the discontinuities of $T_{M E}$, see figure 2 . Additionally, this quantifies the finding that the difference of the mean first passage time between the master equation and the $\Omega$ expansion diminishes with increasing $\mathrm{IP}_{3}$ concentration.

\subsection{Role of fluctuations}

The most important difference between the nonlinear Fokker-Planck equation (11) and van Kampen's expansion (7) is in the diffusion term. It is constant in 

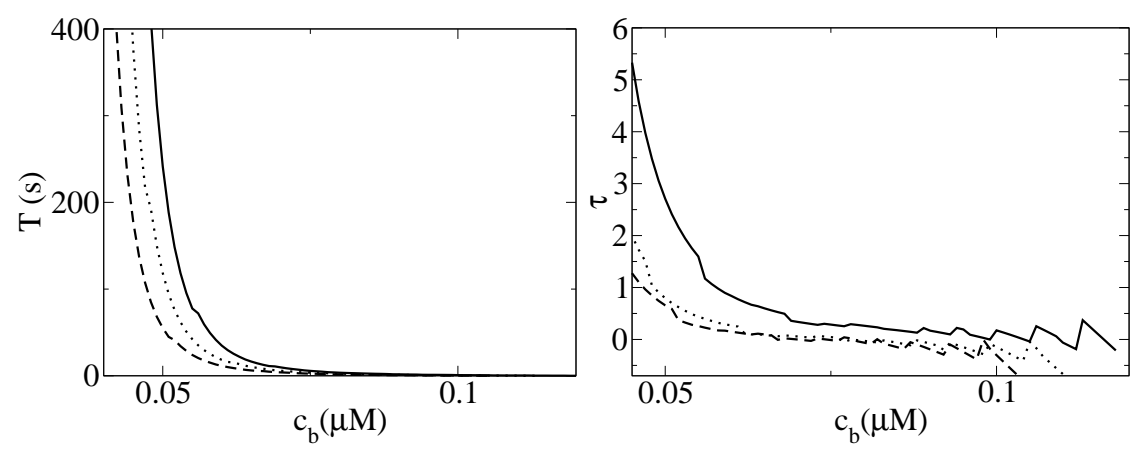

Fig. 3. Mean first passage time for the master equation (left) and the relative difference $\tau:=\left(T_{v K}-T_{M E}\right) / T_{M E}$ of the mean first passage time between van Kampen's method $T_{v K}$ and the master equation $T_{M E}$ (right) in dependence on the base level $c_{b}$ for different values of the $\mathrm{IP}_{3}$ concentration: $I=0.4 \mu \mathrm{M}$ (solid), $0.5 \mu \mathrm{M}$ (dotted), $0.6 \mu \mathrm{M}$ (dashed). Parameter values as in figure 2 and $a_{5}=1(\mu \mathrm{Ms})^{-1}$.

van Kampen's expansion - describing additive noise - and linear in $\phi$ and $\psi$ in the nonlinear Fokker-Planck equation thus describing multiplicative noise. As expected intuitively, the results in figure 2 show a better agreement between the nonlinear Fokker-Planck equation and the master equation than between van Kampen's expansion and the master equation. However, van Kampen's expansion approximates the master equation results rather well for high $\mathrm{IP}_{3}$ and high base level of $\mathrm{Ca}^{2+}$. That is quantified in figure 3. Consequently, additive noise is probably a good approximation in these parameter areas.

\subsection{Distribution of first passage time}

Van Kampen's expansion allows a direct computation of the probability density of the first passage time $\rho(0, t) . \rho(0, t) d t$ is the probability that the absorbing boundary is reached between $t$ and $t+d t$. The starting point of the escape process in the $\Omega$ expansion is $\xi=0$. The $\mathrm{IP}_{3} \mathrm{R}$ cluster is exactly in the macroscopic state $\bar{\phi}$ at $t=0$, so that the noise vanishes at $t=0$. The results for $\rho$ are depicted in figure 4. A convergence of the probability density according to equation (23) requires less than 10 eigenvalues. The curves show the well known rising phase of $\rho$ and the exponential decay. We find a maximal probability that shifts toward shorter times for higher $\mathrm{IP}_{3}$ concentrations. The two graphs in figure 4 illustrate again the influence of the base level. Lowering $c_{b}$ from $60 \mathrm{nM}$ to $40 \mathrm{nM}$ leads to an extreme broadening of the probability distribution and hence to an increase of the mean first passage time (see figure 3 ).

The probability density $\rho$ permits an efficient computation of all moments of $t$ for the escape process. Since the eigenvalues $\lambda_{n}$ and the coefficients $a_{n}$ are known, we immediately arrive at $\left\langle t^{m}\right\rangle=\sum_{n} m ! a_{n} \lambda_{n}^{-m}$ in analogy to equation 

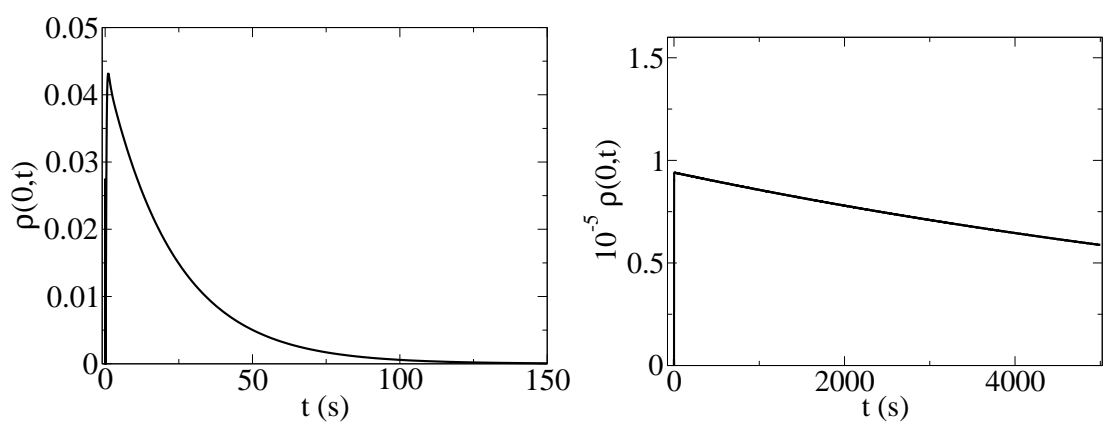

Fig. 4. Probability density $\rho(0, t)$ for van Kampen's expansion. Parameter values as in figure 2 and $I=0.5 \mu \mathrm{M}$. Left panel: $c_{b}=0.06 \mu \mathrm{M}$. Right panel: $c_{b}=0.04 \mu \mathrm{M}$.

(26) due to $v_{n}(0)=1$ for all $n$. The dots in figure 2 depict the results for the variance. The first six eigenvalues suffice for an excellent convergence. That is a direct consequence of the spectrum of the backward Fokker-Planck operator in equation (15).

Figure 5 shows the ratio of the first two eigenvalues $\lambda_{1} / \lambda_{0} . \lambda_{1}$ is only a few times larger than $\lambda_{0}$ for large $\mathrm{IP}_{3}$ and $d_{5}$. However, the ratio increases with decreasing $\mathrm{IP}_{3}$ concentrations and spans more than one order of magnitude for $\mathrm{IP}_{3}$ concentrations smaller than $1 \mu \mathrm{M}$. Hence, already the second term in the expansion (23) is considerably damped in the parameter range in which we are interested $\left(\mathrm{IP}_{3}<1 \mu \mathrm{M}\right)$. Since the eigenvalues constitute a strictly increasing series, i.e. $\lambda_{i}<\lambda_{j}$ for $i<j$, the subsequent terms in the expansion decay even more rapidly. The prominent role of the first term is additionally supported by the expansion coefficients $a_{i}$. The ratio $a_{1} / a_{0}$ is depicted in the right panel of figure 5. It decreases upon lowering the $\mathrm{IP}_{3}$ concentration and tends to zero for very little concentrations. $a_{1}$ is much smaller than $a_{0}$ in parameter ranges where $\lambda_{1} / \lambda_{0} \gg 1$ holds, i.e. where the second term of the series in equation (23) decays much faster than the first one. Consequently, higher terms only contribute marginally in this parameter regime. A detailed analysis of the spectrum and further implications will be provided in an upcoming report.

\subsection{Continuous $\mathrm{Ca}^{2+}$ model}

The results presented so far have been based on a discrete description of the number of open channels. The most important consequence is that the $\mathrm{Ca}^{2+}$ concentration remains constant as long as no channel opens. In the past, investigations on stochastic properties of $\mathrm{IP}_{3} \mathrm{R}$ clusters often employed a continuous model of the ratio of open channels [24, 29, 28]. In these models, the $\mathrm{Ca}^{2+}$ concentration changes even for fractions of open channels corresponding to less than one channel. Therefore, we have analyzed the impact of a continuously modulated number of open channels on the mean first passage 

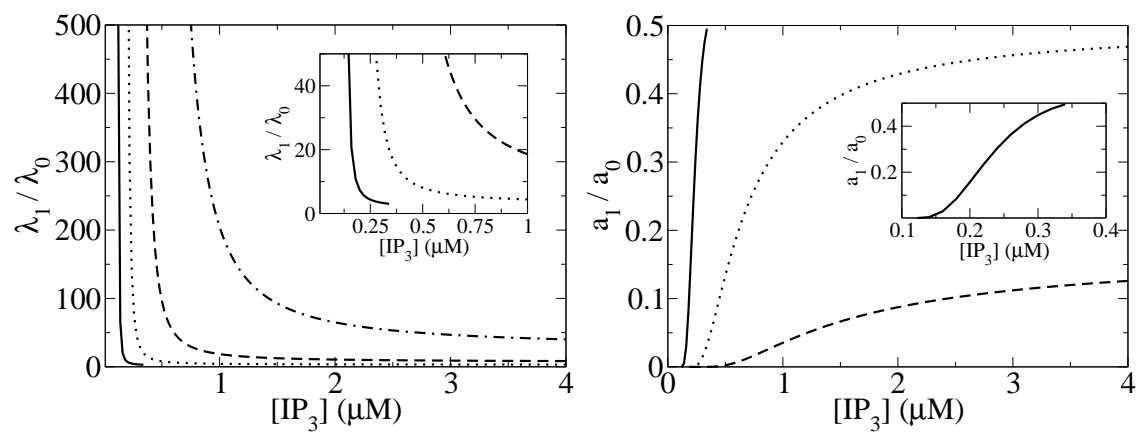

Fig. 5. Ratio of the first two eigenvalues (left panel) and ratio of the first two expansion coefficients (right panel) of equation (23) in dependence on the $\mathrm{IP}_{3}$ concentration. Parameter values as in figure 3 and $d_{5}=0.08234 \mu \mathrm{M}$ (solid), $0.13234 \mu \mathrm{M}$ (dotted), $0.183234 \mu \mathrm{M}$ (dashed) and $0.23234 \mu \mathrm{M}$ (chain-dotted). Insets show a blowup for small $\mathrm{IP}_{3}$ concentrations.

time. The nullclines of the deterministic dynamics for such an ansatz with the same parameter values as before are displayed in the left panel of figure 6 . There is only one stationary state, which is linearly stable for all $\mathrm{IP}_{3}$ concentrations. A prerequisite for a puff is that a sufficient number of subunits can be activated during the escape process from this fixed point. The value of $\psi$ indicates that a large fraction of subunits is inhibited at already moderate $\mathrm{IP}_{3}$ concentrations. It turns out that the remaining fraction of subunits is too low to induce a $\mathrm{Ca}^{2+}$ puff. The high degree of inhibition results from the large $\mathrm{Ca}^{2+}$ fluxes that occur at an open cluster [31]. These fluxes entail $\mathrm{Ca}^{2+}$ concentrations already in the $\mu \mathrm{M}$ range for sizes of the conducting area that are much smaller than that of a single channel. Since these concentrations exceed the dissociation constants for inhibition, most of the subunits are inhibited. Lowering the $\mathrm{IP}_{3}$ concentration does not invoke puffs, either. Although the fraction of inhibited subunits diminishes, the number of subunits that can be activated decreases as well.

We compensate for the elevated $\mathrm{Ca}^{2+}$ concentrations with an increase in the dissociation constant for $\mathrm{Ca}^{2+}$ activation, $d_{5}$. The ensuing nullclines are depicted in the right panel of figure 6 . The left stationary state is linearly stable and corresponds to a low degree of inhibition. The motion of $\phi$ in phase space proceeds along an almost horizontal line through this fixed point during puff initiation. These dynamics are bistable as the potential in figure 7 highlights. $\mathrm{A} \mathrm{Ca}^{2+}$ puff parallels an escape process from the left well over the barrier to the first channel opening.

The time for such an escape process depends on the position of the absorbing boundary with respect to the barrier of the potential. Figure 7 shows the mean first passage time in dependence on the location $\phi$ of this boundary. $\phi$ varies from the value of the potential maximum (see inset) to the value of 

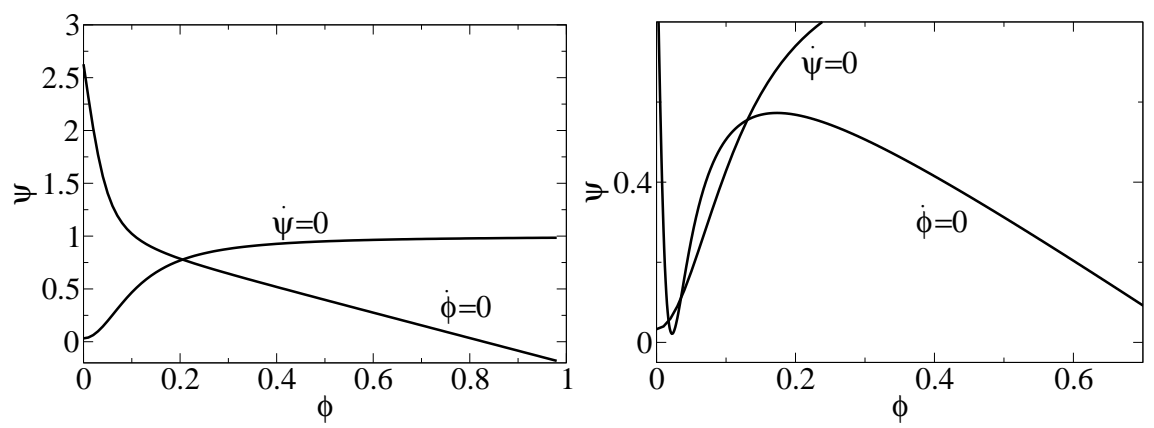

Fig. 6. Nullclines of equation (5). Parameter values as in figure 3 and $k_{l}=$ $0.002 \mathrm{~s}^{-1}, k_{c}=34500 \mathrm{~s}^{-1}, k_{p}=80 \mathrm{~s}^{-1}, E=750 \mu \mathrm{M}, a_{0}=0.03 \mu \mathrm{m}, D=40 \mu \mathrm{m}^{2} \mathrm{~s}^{-1}$, $d_{5}=0.1646 \mu \mathrm{M}, I=0.06 \mu \mathrm{M}$ (left panel), $d_{5}=1.6468 \mu \mathrm{M}, I=0.053 \mu \mathrm{M}$ (right panel).

the first channel opening $\phi_{b}$ (see section 6.1). The steep increase of $T$ for small $\phi$ reflects the influence of the left well. As long as the absorbing boundary is close to the maximum of the potential, reentrance in the left well is possible. That becomes less dominant with increasing $\phi$, so that the mean first passage time reaches the plateau. For the upper range of $\mathrm{IP}_{3}$ concentrations in figure 7 , the value of the plateau equals the mean first passage time. Consequently, the time scale of the puff is set by the properties of the left well. The strong increase of the mean first passage time for smaller $\mathrm{IP}_{3}$ concentrations is due to two reasons. On the one hand, the left well of the bistable potential becomes broader and deeper with lower values of $I$. On the other hand, the absorbing boundary increases in a disproportionally high manner and moves higher on the right branch of the potential.

We exclude van Kampen's expansion in the above analysis, because its validity requires a single stationary state throughout the stochastic motion [34]. In contrast to a constant $\mathrm{Ca}^{2+}$ concentration, the nonlinear Fokker-Planck equation underestimates the results of the master equation. Nevertheless, the results in figure 7 , which correspond to the stochastic fraction of the puff frequency, are in the same range as experimentally determined puff periods [21].

\section{Discussion}

We have derived a master equation and two Fokker-Planck equations for channel cluster behavior in $\mathrm{IP}_{3}$ mediated $\mathrm{Ca}^{2+}$ dynamics. Among the different approaches to approximate a master equation by a Fokker-Planck equation we have chosen van Kampen's $\Omega$ expansion and an ansatz based on the Kramers-Moyal expansion. Master equations and corresponding FokkerPlanck equations for intracellular $\mathrm{Ca}^{2+}$ dynamics have been investigated in 

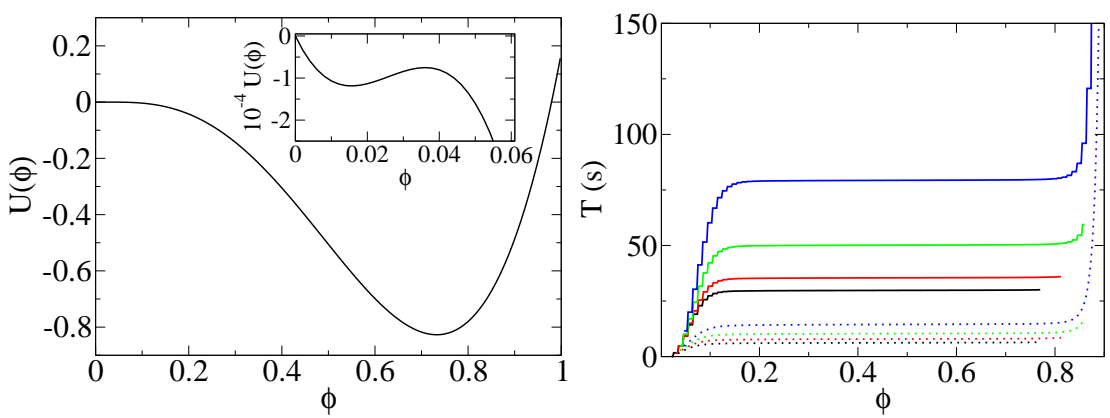

Fig. 7. Left: Potential $U(\phi)$ for $I=0.0483 \mu \mathrm{M}$. The inset depicts an enlarged view for $\phi \approx 0$. Note the difference in scale for the axis. Right (Color): Mean first passage time computed from the master equation (solid lines) and the nonlinear Fokker-Planck equation (dotted lines) for $I=0.0553 \mu \mathrm{M}$ (black), $0.0513 \mu \mathrm{M}$ (red), $0.0473 \mu \mathrm{M}$ (green) and $0.0433 \mu \mathrm{M}$ (blue) in dependence on the position of the absorbing boundary $\phi$. Parameter values as in figure 6, right panel.

the past $[28,29,24]$, but the study at hand is founded on different ideas. Most of the previous contributions employ the Li-Rinzel model [20] for the dynamics of a single subunit of an $\mathrm{IP}_{3}$ receptor. It describes the time evolution of the fraction of subunits that are not inhibited yet, taking advantage of the time scale separation between $\mathrm{IP}_{3}$ activation, $\mathrm{Ca}^{2+}$ activation and $\mathrm{Ca}^{2+}$ inhibition. We have used a state scheme for one subunit that only eliminates the $\mathrm{IP}_{3}$ dynamics adiabatically. It focuses on $\mathrm{Ca}^{2+}$ activation, which is the driving force behind puff initiation. Therefore, we consider $\mathrm{Ca}^{2+}$ activation as the fluctuating variable, whereas $\mathrm{Ca}^{2+}$ inhibition is the random variable in the Li-Rinzel model.

The $\mathrm{Ca}^{2+}$ concentration plays a pivotal role in the initiation of $\mathrm{Ca}^{2+}$ puffs. On the one hand, it fixes the resting state of a cluster, i.e. the starting point of the escape process. On the other hand, it determines the transition rates. The present work has demonstrated that the $\mathrm{Ca}^{2+}$ concentration needs to stay at base level until the first channel opens. Theory provides $\mathrm{Ca}^{2+}$ puffs that are in agreement with experimental results [21] at physiological parameter values only if the $\mathrm{Ca}^{2+}$ concentration remains constant during the entire escape process. These findings underline the discrete character of $\mathrm{IP}_{3} \mathrm{R}$ channels in a cluster [32, 33].

We use a realistic value for the channel flux constant $k_{c}$ in difference to earlier studies [28, 29,24]. That value is based on detailed simulations [31] and leads to $\mathrm{Ca}^{2+}$ concentrations 2-3 orders of magnitude larger than base level at an open channel. That causes models with a continuous number of open channels to fail. The non-vanishing $\mathrm{Ca}^{2+}$ flux at fractions of open channels smaller than 1 resulted in highly elevated $\mathrm{Ca}^{2+}$ concentrations at a cluster due to the large flux density [31]. In turn, that induced a high degree of inhibition. Decreasing the $\mathrm{IP}_{3}$ concentration reduced the level of inhibition, but the 
number of subunits that could be activated decreased, too. The lack of $\mathrm{Ca}^{2+}$ puffs was resolved by increasing the dissociation constant for $\mathrm{Ca}^{2+}$ activation $d_{5}$. The ensuing mean first passage times again complied with experimental results, but at unphysiological values of $d_{5}$. These results demonstrate that parameter values may decide upon the underlying mechanisms. The large $\mathrm{Ca}^{2+}$ fluxes demand a discrete modeling of the $\mathrm{Ca}^{2+}$ release channels. This discrete modelling is one of the aspects of this study setting it apart from previous investigations of stochastic cluster dynamics [24, 28].

At a constant $\mathrm{Ca}^{2+}$ concentration, the main difference between van Kampen's expansion and the nonlinear Fokker-Planck equation is in the character of fluctuations. They correspond to additive noise for the $\Omega$ expansion and to multiplicative noise in the latter approach. Although the noise is intrinsically multiplicative, van Kampen's expansion provides a reasonable approximation, which improves with increasing base level and growing $\mathrm{IP}_{3}$ concentration. It opens up the opportunity for further studies since the $\Omega$ expansion is the only method that yields analytic expressions for the probability density and all higher moments. That distinguishes it from the master equation and the nonlinear Fokker-Planck equation, for which only the first moment is directly accessible.

The dependencies of the mean first passage time on the $\mathrm{Ca}^{2+}$ concentration as well as on the $\mathrm{IP}_{3}$ concentration comply with physiological findings. An increase of the basal $\mathrm{Ca}^{2+}$ concentration enhances the open probability of the $\mathrm{IP}_{3} \mathrm{R}$ channel [4]. Consequently, the mean first passage time is to decrease with growing $\mathrm{Ca}^{2+}$ concentration. Our results fully agree with this activating role of $\mathrm{Ca}^{2+}$ (see figure 3). The same tendency was observed when we increased the $\mathrm{IP}_{3}$ concentration, which agrees with the activating role of $\mathrm{IP}_{3}$.

The present study has provided a framework for a quantitative determination of $\mathrm{Ca}^{2+}$ puff frequencies. The mean first passage times correspond to the stochastic fraction of the inter-puff interval, which is governed by the activation of the $\mathrm{IP}_{3} \mathrm{Rs}$. The second contribution to the inter-puff interval is a deterministic part controlled by puff duration, inhibition and recovery from it. Taking into account that $\mathrm{Ca}^{2+}$ puffs represent the fundamental building blocks of global $\mathrm{Ca}^{2+}$ patterns, our calculations may serve as a starting point to compute periods of $\mathrm{Ca}^{2+}$ waves. Experiments and theoretical studies suggest that the initiation of $\mathrm{Ca}^{2+}$ waves occurs by a nucleation process. Therefore, knowledge of the frequency of $\mathrm{Ca}^{2+}$ puffs is the first step in the calculation of wave frequencies and leads to a deeper understanding of intracellular $\mathrm{Ca}^{2+}$ dynamics.

\section{Appendix A: Combinatorics for subunits}

Measurements on the $\mathrm{IP}_{3}$ receptor have revealed that a minimum number of subunits $h_{m}$ needs to be activated for the channel to open [4]. A single $\mathrm{IP}_{3} \mathrm{R}$ possesses a non zero open probability only if at least $h_{m}$ subunits are in the 
state 10. Activation in the cell occurs of course for a subunit already associated with a certain receptor. With our model, the number of open channels depends on the arrangement of $n_{10}$ activatable subunits on the receptors. Here, we derive the distribution of open channels resulting from such a random scattering of activatable subunits and its properties, whereas the mean was used earlier. To this aim we consider $N$ receptors with $h$ subunits each. Let $n_{i}, i=1, \ldots, h$ denote the number of receptors with $i$ activatable subunits, then the number of possible configurations for a given set $\left\{n_{i}\right\}:=\left\{n_{1}, \ldots, n_{h}\right\}$ that satisfies

$$
n_{0}+\ldots+n_{h}=N, \quad n_{1}+2 n_{2}+\ldots+h n_{h}=n_{10}
$$

is

$$
M\left(\left\{n_{i}\right\}\right):=\frac{N !}{n_{0} ! \cdots n_{h} !}\left(\begin{array}{c}
h \\
0
\end{array}\right)^{n_{0}}\left(\begin{array}{l}
h \\
1
\end{array}\right)^{n_{1}} \cdots\left(\begin{array}{l}
h \\
h
\end{array}\right)^{n_{h}} .
$$

The fraction represents the number of permutations for the set $\left\{n_{i}\right\}$, whereas the binomial coefficients take into account the number of ways how to distribute $i$ activatable subunits on a single receptor. The total number of configurations is given by

$$
\Gamma:=\sum_{\left\{n_{i}\right\}}^{\star} M\left(\left\{n_{i}\right\}\right)
$$

The asterisk indicates the summation with the restrictions of equation (35). To evaluate equation (37), we introduce a generating function

$$
f_{1}(z):=\sum_{\left\{n_{i}\right\}}^{\prime} M\left(\left\{n_{i}\right\}\right) z^{l}, \quad l=n_{1}+\ldots+h n_{h} .
$$

The prime refers to the restriction $n_{0}+\ldots+n_{h}=N$. Therefore, the total number of configurations follows from the generating function as

$$
\Gamma=\left.\frac{1}{n_{10} !} \frac{d^{n_{10}}}{d z^{n_{10}}} f_{1}(z)\right|_{z=0} .
$$

Due to the identity

$$
\begin{aligned}
f_{1}(z) & =\sum_{n_{i}=0}^{N} \frac{N !}{n_{0} ! \cdots n_{h} !}\left(\begin{array}{l}
h \\
0
\end{array}\right)^{n_{0}}\left[\left(\begin{array}{l}
h \\
1
\end{array}\right) z^{1}\right]^{n_{1}} \cdots\left[\left(\begin{array}{l}
h \\
h
\end{array}\right) z^{h}\right]^{n_{h}} \\
& =\left[\left(\begin{array}{l}
h \\
0
\end{array}\right)+\ldots+\left(\begin{array}{l}
h \\
h
\end{array}\right) z^{h}\right]^{N}=(1+z)^{h N}=\sum_{j=0}^{h N}\left(\begin{array}{c}
h N \\
j
\end{array}\right) z^{j},
\end{aligned}
$$

we finally arrive at $\Gamma=\left(\begin{array}{l}h N \\ n_{10}\end{array}\right)$, which complies with the combinatorics of choosing $n_{10}$ subunits from a total of $h N$ subunits. Consequently, the probability distribution of $n_{j}$ for a fixed value of $j \in\{0, \ldots, h\}$ is given by 


$$
\begin{aligned}
p\left(n_{j}\right) & =\frac{1}{\Gamma} \sum_{\substack{\left\{n_{i}\right\} \\
i \neq j}}^{\star} \frac{N !}{n_{0} ! \cdots n_{h} !}\left(\begin{array}{c}
h \\
0
\end{array}\right)^{n_{0}}\left(\begin{array}{l}
h \\
1
\end{array}\right)^{n_{1}} \cdots\left(\begin{array}{l}
h \\
h
\end{array}\right)^{n_{h}} \\
& =\frac{1}{\Gamma}\left(\begin{array}{c}
N \\
n_{j}
\end{array}\right)\left(\begin{array}{c}
h \\
j
\end{array}\right)^{n_{j}} \sum_{\substack{\left\{n_{i}\right\} \\
i \neq j}}^{\star}\left(N-n_{j}\right) ! \prod_{\substack{l=0 \\
l \neq j}}^{h} \frac{1}{n_{l} !}\left(\begin{array}{c}
h \\
l
\end{array}\right)^{n_{l}} .
\end{aligned}
$$

Equation (41) is most conveniently computed as

$$
p\left(n_{j}\right)=\left.\frac{1}{\Gamma}\left(\begin{array}{c}
N \\
n_{j}
\end{array}\right)\left(\begin{array}{c}
h \\
j
\end{array}\right)^{n_{j}} \frac{1}{n_{10} !} \frac{d^{n_{10}}}{d z^{n_{10}}} f_{2}(z)\right|_{z=0},
$$

where we used the generating function

$$
\begin{aligned}
f_{2}(z) & :=\sum_{\substack{\left\{n_{i}\right\} \\
i \neq j}}^{\prime} \tilde{N} ! \prod_{\substack{l=0 \\
l \neq j}}^{h} \frac{1}{n_{l} !}\left[\left(\begin{array}{l}
h \\
l
\end{array}\right) z^{l}\right]^{n_{l}} \\
& =\sum_{i=0}^{\tilde{N}} \sum_{l=0}^{h i}\left(\begin{array}{c}
\tilde{N} \\
i
\end{array}\right)\left(\begin{array}{c}
h i \\
l
\end{array}\right)\left[-\left(\begin{array}{c}
h \\
j
\end{array}\right)\right]^{\tilde{N}-i} z^{l+j(\tilde{N}-i)} .
\end{aligned}
$$

Here, the prime denotes the restriction

$$
n_{0}+\ldots+n_{j-1}+n_{j+1}+\cdots+n_{h}=N-n_{j}=: \tilde{N} .
$$

In the case $j=0$ the derivatives in equation (42) can be performed explicitly, so that

$$
p\left(n_{0}\right)=\frac{1}{\Gamma}\left(\begin{array}{c}
N \\
n_{0}
\end{array}\right) \sum_{j=0}^{\tilde{N}}\left(\begin{array}{c}
\tilde{N} \\
j
\end{array}\right)\left(\begin{array}{c}
j h \\
n_{10}
\end{array}\right)(-1)^{\tilde{N}-j} .
$$

The above analysis remains valid, when we interchange the number of activatable subunits $n_{10}$ and the number of the remaining $N h-n_{10}$ subunits. Such a transition corresponds to the exchange of balls and voids in classical combinatorics. In that picture, equation (45) would represent the probability distribution of fully occupied receptors, i.e.

$$
p\left(n_{h}\right)=\frac{1}{\Gamma}\left(\begin{array}{c}
N \\
n_{h}
\end{array}\right) \sum_{j=0}^{\tilde{N}}\left(\begin{array}{c}
\tilde{N} \\
j
\end{array}\right)\left(\begin{array}{c}
j h \\
N h-n_{10}
\end{array}\right)(-1)^{\tilde{N}-j} .
$$

Equation (46) arises from equation (45) by substituting $n_{10}$ by $N h-n_{10}$ and $n_{0}$ by $n_{h}$.

To gain further insight into the probability distributions we calculate the first two moments. For the average we start with

$$
\left\langle n_{j}\right\rangle=\frac{1}{\Gamma} \sum_{\left\{n_{i}\right\}}^{\star} n_{j} M\left(\left\{n_{i}\right\}\right)
$$


because a closed expression for the probability distribution is only available for the two cases presented above. Defining the corresponding generating function

$$
f_{3}(z):=\frac{1}{\Gamma} \sum_{\left\{n_{i}\right\}}^{\prime} n_{j} M\left(\left\{n_{i}\right\}\right) z^{l}, \quad l=n_{1}+\ldots+h n_{h}
$$

we find

$$
\left\langle n_{j}\right\rangle=\left.\frac{1}{n_{10} !} \frac{d^{n_{10}}}{d z^{n_{10}}} f_{3}(z)\right|_{z=0}=\frac{N}{\Gamma}\left(\begin{array}{c}
h \\
j
\end{array}\right)\left(\begin{array}{c}
h(N-1) \\
n_{10}-j
\end{array}\right) .
$$

In the limit $N \rightarrow \infty, n_{10} \rightarrow \infty$ we recover the result from [3]. Analogously evaluation of the second moments results in

$$
\left\langle n_{l} n_{k}\right\rangle=\frac{N(N-1)}{\Gamma}\left(\begin{array}{l}
h \\
l
\end{array}\right)\left(\begin{array}{l}
h \\
k
\end{array}\right)\left(\begin{array}{c}
h(N-2) \\
n_{10}-l-k
\end{array}\right)+\delta_{k, l} \frac{N}{\Gamma}\left(\begin{array}{c}
h \\
l
\end{array}\right)\left(\begin{array}{c}
h(N-1) \\
n_{10}-l
\end{array}\right) .
$$

Applying these general expressions to $\mathrm{IP}_{3} \mathrm{Rs}$ requires values for $h, h_{m}$ and $N$. The tetrameric structure of the receptor ensues $h=4$. However, previous results by different groups are based on $h=3$. We therefore compute the statistics for both cases. Experiments on a single channel have shown four conductance levels, each a multiple of $20 \mathrm{pS}$, with a predominance of opening to the third level $[4,35]$. Thus, we set $h_{m}=3$. The number of receptors in a cluster has not been measured yet. We employ $N=25$ following recent estimates by Swillens and Dupont [30].

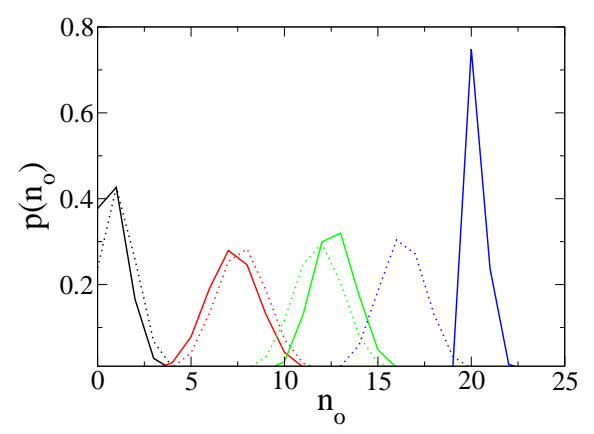

Fig. 8. (Color) Probability distribution $p\left(n_{o}\right)$ for $n_{o}=n_{3}, h=3$ (solid) and $n_{o}=$ $n_{3}+n_{4}, h=4$ (dotted) for $N=25$ and different $n_{10}$. Values of $n_{10}$ are 25 (black), 50 (red), 60 (green) and 70 (blue).

The probability distributions $p\left(n_{3}+n_{4}\right)$ with $h=4$ and $p\left(n_{3}\right)$ with $h=3$ are depicted in figure 8 . They both agree very well. This is also supported by their mean and variance as shown in figure 9 . In the left panel we also include the position of the maxima of the distributions indicated by dots. They closely follow the average. Due to the narrowness of the distributions demonstrated 
by the small variance as well as the accordance between the mean and the maximum we calculate the number of open channels $n_{c}$ from the average for a given value of $n_{10}$ :

$$
\begin{aligned}
n_{a}^{(3)} & =N r^{3} \frac{n_{10}}{3 N} \frac{n_{10}-1}{3 N-1} \frac{n_{10}-2}{3 N-2} \\
n_{a}^{(3,4)} & =N r^{3} \frac{n_{10}}{4 N} \frac{n_{10}-1}{4 N-1} \frac{n_{10}-2}{4 N-2}\left[\frac{n_{10}-3}{4 N-3}(4-3 r)+4\left(1-\frac{n_{10}}{4 N}\right)\right]
\end{aligned}
$$

Here $r:=I /\left(I+d_{1}\right)$ denotes the fraction of subunits in the activatable state 10 that are activated. The subscripts $(3)$ and $(3,4)$ indicate that we used $p\left(n_{3}\right), h=3$ and $p\left(n_{3}+n_{4}\right), h=4$ for averaging, respectively. Note that in the limit $N \rightarrow \infty, n_{10} \rightarrow \infty$ equations (51), (52) reduce to the well known expressions of the deterministic description. All results in section 6 are based on equation (52), which can be further simplified by approximating all denominators by $4 N$ due to $4 N \gg 1$.
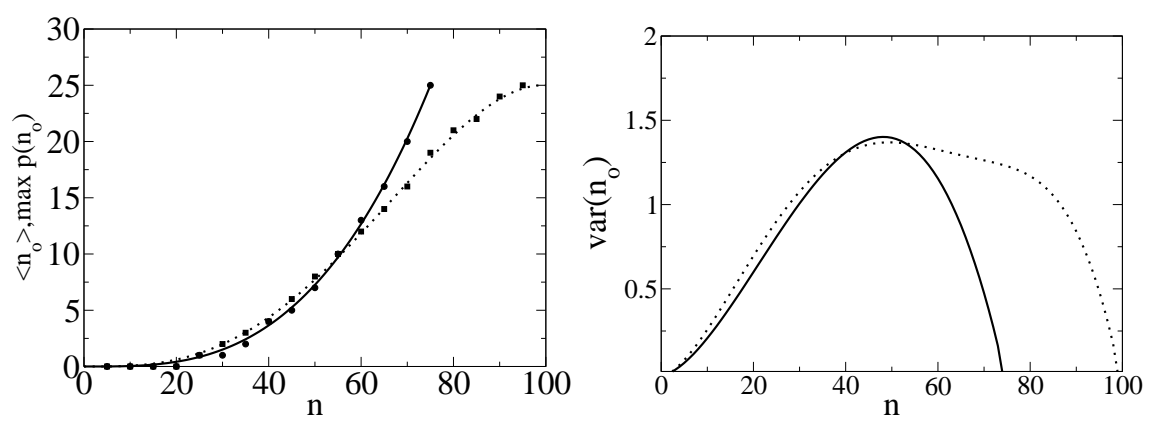

Fig. 9. Mean (left) and variance (right) of $n_{o}$ for $n_{o}=n_{3}, h=3$ (solid) and $n_{o}=n_{3}+n_{4}, h=4$ (dotted). The left panel shows the position of $\max p\left(n_{o}\right)$ as dots and scares, respectively.

\section{Appendix B: Proof of equation (32)}

This section deals with the proof of equation (32). It is based on the identity

$$
\sum_{k=0}^{j}\left(\begin{array}{l}
j \\
k
\end{array}\right) \frac{(-1)^{k}}{2 k+1}=\frac{2^{2 j}(j !)^{2}}{(2 j+1) !}
$$

which we now proof. We transform the left hand side of equation (53) according to 


$$
\sum_{k=0}^{j}\left(\begin{array}{l}
j \\
k
\end{array}\right)(-1)^{k} \int_{0}^{1} t^{2 k} d t=\int_{0}^{1} \sum_{k=0}^{j}\left(\begin{array}{l}
j \\
k
\end{array}\right)\left(-t^{2}\right)^{k} d t=\int_{0}^{1}\left(1-t^{2}\right)^{j} d t
$$

It can be simplified with Euler's Beta function $B(z, w)$. From its definition

$$
B(z, w):=\int_{0}^{1} t^{z-1}(1-t)^{w-1} d t
$$

follows

$$
\int_{a}^{b}(t-a)^{z-1}(b-t)^{w-1} d t=(b-a)^{z+w-1} B(z, w) .
$$

Hence we express the integral in equation (54) through

$$
\int_{0}^{1}\left(1-t^{2}\right)^{j} d t=\frac{1}{2} \int_{-1}^{1}(t+1)^{j}(1-t)^{j} d t=2^{2 j} B(j+1, j+1) .
$$

According to [1] the Beta function is related to the Gamma function $\Gamma(z)$ via $B(z, w)=\Gamma(z) \Gamma(w) / \Gamma(z+w)$, so that we find

$$
\sum_{l=0}^{j}\left(\begin{array}{l}
j \\
l
\end{array}\right) \frac{(-1)^{l}}{2 l+1}=2^{2 j} \frac{\Gamma(j+1)^{2}}{\Gamma(2 j+2)}=\frac{2^{2 j}(j !)^{2}}{(2 j+1) !}
$$

due to $n !=\Gamma(n+1)$, which proofs equation (53). Expanding the right hand side yields

$$
\frac{2^{2 j}(j !)^{2}}{(2 j+1) !}=\frac{2 \cdot 1}{2} \cdot \frac{2}{3} \cdot \frac{2 \cdot 2}{4} \cdot \frac{2}{5} \cdot \frac{2 \cdot 3}{6} \cdots \frac{2 \cdot j}{2 j} \cdot \frac{2}{2 j+1} j !=\frac{j !}{\left(\frac{3}{2}\right)_{j}} .
$$

This proofs equation (32) when we use $j !=(1)_{j}$.

\section{References}

1. M. Abramowitz and I. Stegun, editors. Handbook of mathematical functions. Dover Publications, New York, 1974.

2. Jerry M. Adams and Suzanne Cory. The bcl-2 protein family: Arbiters of cell survival. Science, 281(5381):1322-1326, 1998.

3. M. Bär, M. Falcke, H. Levine, and L. S. Tsimring. Discrete stochastic modeling of calcium channel dynamics. Phys. Rev. Lett., 84:5664-5667, 2000.

4. I. Bezprozvanny, J. Watras, and B.E. Ehrlich. Bell-shaped calcium-response curves of $\operatorname{Ins}(1,4,5) \mathrm{P}_{3}$ - and calcium-gated channels form endoplasmic reticulum of cerebellum. Nature, 351:751-754, 1991. 
5. G.W. De Young and J. Keizer. A single inositol 1,4,5-triphosphate-receptorbased model for agonist-stimulated oscillations in $\mathrm{Ca}^{2+}$ concentration. Proc. Natl. Acad. Sci. USA, 89:9895-9899, 1992.

6. M. Falcke. On the role of stochastic channel behavior in intracellular $\mathrm{Ca}^{2+}$ dynamics. Biophys. J., 84:42-56, 2003.

7. M. Falcke. Reading the pattern in living cells - the physics of $\mathrm{Ca}^{2+}$ signaling. Advances in Physics, 53:255-440, 2004.

8. C.W Gardiner. Handbook of stochastic methods. Springer, Berlin, 3 edition, 2004.

9. M. Gitterman and G.H. Weiss. Some comments on approximations to the master equation. Physica A, 170:503-510, 1991.

10. A. Goldbeter. Biochemical Oscillations and Cellular Rhythms. Cambridge University Press, Cambridge, 1996.

11. H. Grabert, P. Hänggi, and I.Oppenheim. Fluctuations in reversible chemical reactions. Physica, 117A:300-316, 1983.

12. P. Hänggi, H. Grabert, P. Talkner, and H. Thomas. Bistable systems: Maser equation versus Fokker-Planck modelling. Phys. Rev. A, 29:371-378, 1984.

13. H. Henry and H. Levine. Wave nucleation in excitable systems in the low noise limit. Phys. Rev. E, 68:031914-1-5, 2003.

14. B. Hille. Ion channels of excitable membranes. Sinauer Assoicates, Inc. Publishers, Sunderland, MA USA, 3rd edition, 2001.

15. Roger A. Horn and Charles R. Johnson. Matrix Analysis. Cambridge University Press, Cambridge, 1999.

16. M. Howard and A. D. Rutenberg. Pattern formation inside bacteria: Fluctuations due to low copy number of proteins. Phys. Rev. Let., 90:128102-1-4, 2003.

17. J. Keener and J. Sneyd. Mathematical physiology. Springer, New York, 1998.

18. H.A. Kramers. Brownian motion in a field of force and the diffusion model of chemical reactions. Physica, 7:284-304, 1940.

19. MD Levin, CJ Morton-Firth, WN Abouhamad, RB Bourret, and D Bray. Origins of individual swimming behavior in bacteria. Biophysical Journal, 74(1):175-181, 1998.

20. Y. Li and J. Rinzel. Equations for $\mathrm{InsP}_{3}$ receptor-mediated $\left[\mathrm{Ca}^{2+}\right]_{i}$ oscillations derived from a detailed kinetic model: A hodgkin-huxley like formalism. $J$. theor. Biol., 166:461-473, 1994.

21. J.S. Marchant and I. Parker. Role of elementary $\mathrm{Ca}^{2+}$ puffs in generating repetitive $\mathrm{Ca}^{2+}$ oscillations. EMBO J., 20:65-76, 2001.

22. Mark P. Mattson. Pathways towards and away from alzheimer's disease. Nature, 430(7000):631-639, 2004

23. Harley H. McAdams and Adam Arkin. Stochastic mechanisms in gene expression. Proceedings of the National Academy of Sciences, 94(3):814-819, 1997.

24. L. Meinhold and L. Schimansky-Geier. Analytical description of stochastic calcium-signal periodicity. Phys. Rev. E, 66:050901(R)-1-4, 2002.

25. J.E. Moyal. Stochastic processes and statistical physics. J. Roy. Stat. Soc. B, 11:150-210, 1949.

26. Christopher V. Rao, Denise M. Wolf, and Adam P. Arkin. Control, exploitation and tolerance of intracellular noise. Nature, 420:231-237, 2002.

27. H. Risken. The Fokker-Planck equation. Springer, Berlin, 1984.

28. J. W. Shuai and P. Jung. Optimal intracellular calcium signalling. Phys. Rev. Lett., 88(6):068102-1-4, 2002. 
29. J. W. Shuai and P. Jung. Stochastic properties of $\mathrm{Ca}^{2+}$ release of inositol 1,4,5trisphosphate receptor clusters. Biophys. J., 83(1):87-97, 2002.

30. S. Swillens, G. Dupont, L. Combettes, and P. Champeil. From calcium blips to calcium puffs: Theoretical analysis of the requirements for interchannel communication. Proc. Natl. Acad. Sci. USA, 96:13750-13755, 1999.

31. R. Thul and M. Falcke. Release currents of $\mathrm{IP}_{3}$ receptor channel clusters and concentration profiles. Biophys. J., 86:2660-2673, 2004.

32. R. Thul and M. Falcke. Stability of membrane bound reactions. Phys. Rev. Lett., 93:188103-1-4, 2004.

33. R. Thul and M. Falcke. Reactive clusters on a membrane. Phys. Biol., 2:51-59, 2005.

34. N.G. van Kampen. Stochastic processes in physics and chemistry. NorthHolland, Amsterdam, 2001.

35. J. Watras, I. Bezprozvanny, and B. Ehrlich. Inositol 1,4,5-trisphosphate-gated channels in cerebellum: presence of multiple conductance states. J. Neurosci., 11:3239-3245, 1991. 


\section{Index}

$\mathrm{Ca}^{2+}$ puff, 3

$\mathrm{IP}_{3}$ receptor, 2

De Young Keizer model, 4, 5

Fokker-Planck equation, 7
Master equation, 5, 6, 9

Mean first passage time, 14

nonlinear FPE, 13

Master equation, 14

van Kampen's expansion, 12 
